\title{
A STUDY OF THE CONTINUA OF THE NUCLEI OF GALAXIES*
}

\author{
D. ALLOIN, Y. ANDRILLAT and S. SOUFFRIN
}

Institut d'Astrophysique, Paris, France and Faculté des Sciences, Montpellier, France

\begin{abstract}
Many people have attacked the problem of synthesizing the stellar population of the galaxies. We have performed such a synthesis, by using only the intensities of absorption lines in the nuclei of galaxies. It is then possible to obtain a synthesized continuous spectrum even if there are several possible solutions for the stellar composition, and the computed continuum does not vary much with the assumed model of stellar population.

The method is to fit the equivalent widths of the absorption lines using different stellar compositions. In order to avoid any instrumental effect, we have observed different lines and bands from 3500 to $8500 \AA$ in stars of various well-known stellar types and luminosity classes, under the same conditions as the galaxies.

We have applied this method to some 'ordinary' nuclei and to some Seyfert ones. As an example, we show the results for M81 and for NGC 1068.
\end{abstract}

\section{M81}

The first result concerns the stellar population: as was already noticed by Spinrad and Wood, we find an important contribution by $\mathrm{M}$ dwarfs (40 to $50 \%$ of the visible continuum) and also by $\mathrm{K}$ stars $(30 \%)$. It is, therefore, likely that this nucleus has a very strong infrared spectrum.

The results for the continuum spectrum are shown in Figure 1.

The observed continuum does not show any reddening, when compared to the computed one. The lack of data concerning the continuum of M stars beyond $7000 \AA$

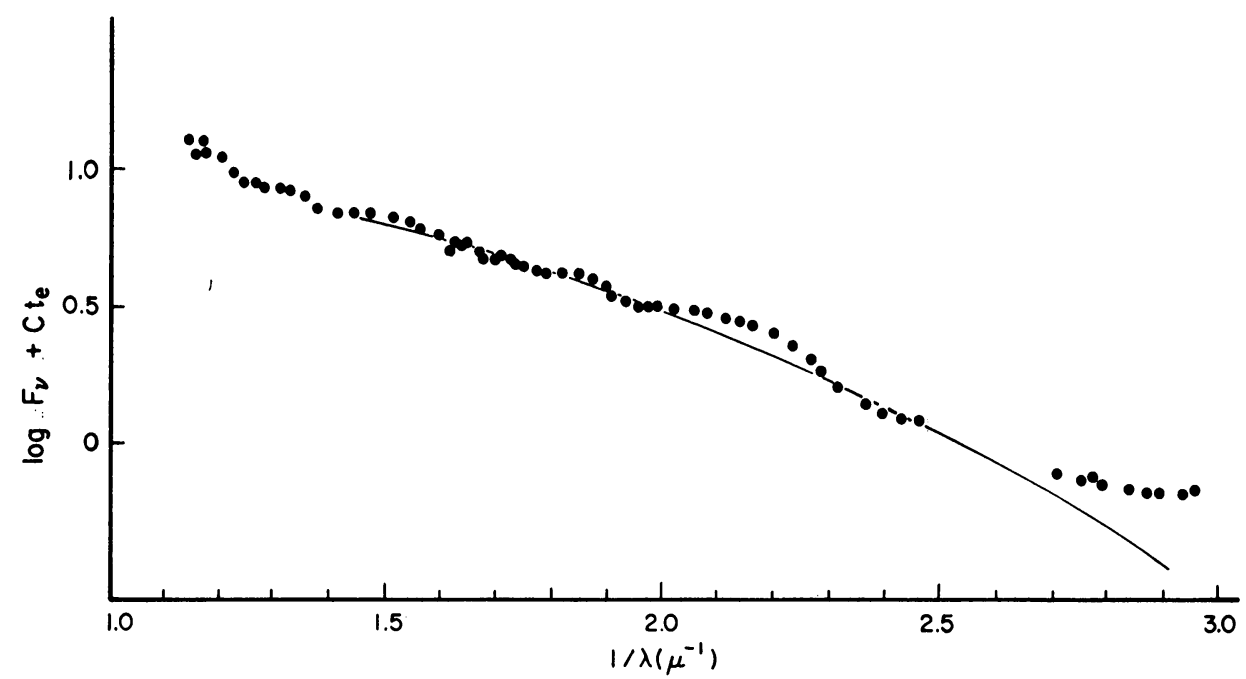

Fig. 1. The continuum energy distribution in the optical spectrum of the nucleus of $M 81$.

* The observations were performed at the Haute-Provence Observatory (CNRS). 
prevents us from computing the synthetic continuum in the near infrared. However, we can conclude from a study of the CaII 8542 line that there is no important nonstellar contribution at wavelength $8500 \AA$. Finally, the figure shows the presence of a non-stellar continuum in the ultraviolet range, which can be identified with the thermal emission of the gas.

The conclusions of this study are as follows:

there is no reddening other than that due to the tilt of the plane of the galaxy; there is no infrared non-thermal continuum;

the thermal Balmer continuum comes from the gas.

\section{NGC 1068}

In the Seyfert nuclei, we have taken into account the possibility of a non-stellar component in the visible range by adding 'fictitious' stars contributing to the continuum with a given spectral distribution, but not contributing to the lines. For NGC 1068 the spectral distribution of the non-thermal continuum has been deduced from published polarization measurements by Visvanathan and Oke, and the spectral distribution of the thermal emission has been corrected for the same amount of reddening that Wampler has found for the emission lines.

One can then obtain a computed continuum fitting the observed one, with a very cold stellar population ( $\mathrm{K}$ and $\mathrm{M}$ stars). The stellar continuum does not show any reddening; hence, the reddening of the emission lines should come from a small region of the nucleus, probably filamentary clouds ejected from the nucleus and containing dust, as Hoyle and Wickramasinghe have suggested.

Two papers relevant to the subject should appear shortly in Astron. Astrophys.

\section{Discussion}

de Vaucouleurs: How many absorption lines did you measure for equivalent width, and how many stellar spectral types did you use in the population fit?

Mme Souffrin: So far we have measured seven lines: CaII 23933 , CaII + HI 23968 , CaI 24227 , G-band, MgI triplet, $\mathrm{Na} \mathrm{D}$ lines, and CaII 28542 . We have used about five spectral types in the population fit.

King: Spinrad has also observed M81, at many line and continuum wavelengths, and also concludes that the population of the center includes many $\mathrm{K}$ and $\mathrm{M}$ dwarfs. Minkowski and $\mathrm{I}$ have included $M 81$ in our velocity dispersion and photometry program, and preliminary results indicate a high $M / L$. 